\title{
Influence of packing on the vibrational properties of infinite and finite bundles of carbon nanotubes
}

\author{
Luc Henrard, ${ }^{1}$ Valentin N. Popov, ${ }^{2, *}$ and Angel Rubio ${ }^{3}$ \\ ${ }^{1}$ Laboratoire de Physique du Solide, Facultés Universitaires Notre-Dame de la Paix, 5000 Namur, Belgium \\ ${ }^{2}$ Physics Department, University of Antwerp (RUCA), 2020 Antwerp, Belgium \\ ${ }^{3}$ Laboratoire de Solides Irradiés (LSI), CNRS-CEA, École Polytechnique, F-91128 Palaiseau, France; \\ and Departamento de Física de Materiales, Facultad de Ciencias Químicas, Universidad del Pais Vasco/Euskal Herriko Unibertsitatea, \\ Apartado Postal 1072, 20018 San Sebastián/Donostia, Basque Country, Spain; \\ and Donostia International Physics Center (DIPC) and Centro Mixto CSIC-UPV/EHU, 20018 San Sebastián, Spain
}

(Received 28 February 2001; revised manuscript received 30 July 2001; published 19 October 2001)

\begin{abstract}
The quantitative analysis of the vibrational properties of carbon nanotubes is a key issue for the interpretation of Raman experiments. In particular, a reliable characterization of the atomic structure of single-wall carbon nanotubes produced under various conditions is mainly based on the interpretation of low-frequency (100-300 $\mathrm{cm}^{-1}$ ) Raman spectra. In the present work, we analyze the influence of the packing of the tubes on these low-frequency modes. We find that the low-frequency spectra of crystals of single-wall carbon nanotubes present two intense Raman modes instead of a single fully symmetric $A_{1 g}$ mode characteristic of isolated tubes. The second mode has a non-negligible intensity for crystals formed with nanotubes of radii larger than $7 \AA$. For finite number of tubes in a bundle, two breathinglike intense modes appear as a specific signature. Finally, our simulation for inhomogenous bundles made of a large number of tubes does not reveal any specific signature of the individual tubes in the low-frequency Raman spectra.
\end{abstract}

DOI: 10.1103/PhysRevB.64.205403

PACS number(s): 61.46.+w, 63.22.+m, 78.30.-j

\section{INTRODUCTION}

The production of bulk quantities of single-wall carbon nanotubes (SWNTs) is now routinely achieved by different methods (Ref. 1). However, the growth mechanism itself is still not well understood or controlled. This means that, for the time being, a specific nanotube (with definite diameter and/or chirality) cannot be produced or selected at will. Furthermore, the development of reliable, fast, and accurate characterization tools is still a major point of study. In particular, high-resolution transmission electron microscope and electron diffraction give semilocal quantitative information on tube atomic structures averaged on one bundle; ${ }^{2}$ global $\mathrm{x}$-ray and neutron diffraction allow a determination of the mean hexagonal packing parameter of a macroscopic SWNT sample; ${ }^{3}$ and local scanning tunneling microscopy-scanning tunneling spectroscopy lead to a precise determination of the wrapping indices of SWNT (Refs. 4 and 5) but cannot be considered as routine experiments to control sample production.

One of the most widespread techniques for the analysis of carbon nanotubes is undeniably Raman spectroscopy. ${ }^{6,7} \mathrm{Be}-$ side the modes that have a direct counterpart in graphite (the C-C stretching) at high frequency $\left(1550-1600 \mathrm{~cm}^{-1}\right)$, the low-frequency modes $\left(100-300 \mathrm{~cm}^{-1}\right)$ appear to be characteristic of closed-shell carbon compounds. One of them, the totally symmetric $A_{1 g}$ mode, also called the radial breathing mode (BM or RBM), has been the object of many experimental and theoretical studies because of its highexcitation cross section and resonant behavior. The first theoretical investigations within a force-constant approach have led to a linear dependence of the frequency of the breathing mode with the inverse tube radius $r, \omega=C / r$ with
$C=1170 \mathrm{~cm}^{-1} \AA$ (Ref. 8) or $C=1147 \mathrm{~cm}^{-1} \AA$ (Ref. 9). This conclusion has been revisited by means of methods that account for the rehybridization of the C-C bond during the vibration and a slight chirality dependence has been deduced $\left[C_{(n, n)}=1180 \mathrm{~cm}^{-1} \AA\right.$ and $C_{(n, 0)}=1160 \mathrm{~cm}^{-1} \AA$ by ab initio local-density approximation in the density-functional theory ${ }^{10,11}$ and $C_{(n, n)}=1301 \mathrm{~cm}^{-1} \AA$ and $C_{(n, 0)}$ $=1282 \mathrm{~cm}^{-1} \AA$ by a nonorthogonal tight-binding (TB) approach $^{12}$.

However, the direct comparison of these values to experimental data remains problematic due to the following reasons:

(i) The Raman scattering from carbon nanotubes is a resonant process due to the coincidence of the incident laser frequency and the electronic van Hove singularity transitions. A combined experimental and simulation analysis of these effects for the BM of an ensemble of tubes has been recently proposed. ${ }^{13}$

(ii) SWNTs turn out to be most often packed into hexagonal microcrystals of several tens of individuals. The effects of intertube interactions on BM have then to be carefully worked out before a comparison to experimental results could be made properly.

Infinite crystals of nanotubes have been considered in the tight-binding-Lennard-Jones (LJ) model by several authors. Venkateswaran et al. $^{14}$ found a $8 \%$ upshift of the $(9,9)$ - (we use the Hamada indices ${ }^{15}$ throughout this paper) tube BM when bundled; Kahn and $\mathrm{Lu}^{16}$ reported a $6 \%$ increase for a $(10,10)$-tube bundle. In a more exhaustive data set, we found $^{12}$ a $10 \%$ (11\%) upshift for $(9,9)[(10,10)]$ bundles and that this shift ranges from $5 \%$ for tubes with $r=3.45 \AA$ to $15 \%$ for $r=8.21 \AA$. The differences between these results stem from different parametrization for the tight-binding model and Lennard-Jones potential and, in the case of Ref. 
16 , by the fact that the system was not relaxed to its minimum of potential. More fundamentally, the imposed radial motion of $\mathrm{C}$ atoms (Ref. 12) leads to an overestimation of the packing effect as discussed in Sec. IV A. On the experimental side, Kataura et al. ${ }^{17}$ succeeded to pass round the fact that macroscopic quantities of isolated SWNTs are not available by using bromine-doped nanotubes and found a $4 \%$ increase of BM frequency when tubes are bundled. Furthermore, recent surface-enhanced-Raman-spectroscopy (SERS) on nanotubes deposited on metallic substrate ${ }^{18-22}$ or nanotubes in contact with silver colloidal cluster ${ }^{23}$ opened the possibility to study individual clusters made of a few nanotubes.

In the present paper, we investigate the role of nanotube packing by the following.

(i) Using a continuum approach of the LJ pair potential to investigate the intertube modes at frequencies lower than $100 \mathrm{~cm}^{-1}$ for finite and infinite bundles. These modes have been proposed to play an important role in the temperature dependence of the bundle specific heat. ${ }^{24,25}$

(ii) Considering the low-frequency Raman active modes of isolated and bundled tubes. We found that tubes larger than $(10,10)$ are rather deformable and can no longer be considered as rigid tubes. We have analyzed the influence of this possibility of deformation on the intensities of modes that derive from the BM of isolated SWNT. We conclude that the breathinglike mode deviates from the pure radial pattern for large radii and that this effect becomes significant for tube radii larger than $7 \AA$. Moreover, for infinite bundles, a second mode with predominant breathinglike character appears at higher frequency $\left[197 \mathrm{~cm}^{-1}\right.$ for $(12,12)$ bundle].

(iii) Bundles made of a finite number of tubes are considered. The cross-sectional size of the bundles depends on the experimental methods and conditions and are far from being regular in one production batch. We have then considered of prime interest to investigate the transition from the isolatedtube case to the infinite crystals. For bundles made of tubes with $r<6 \AA$, the tubes could be considered as rigid breathing entities and the in-phase breathing mode dominates the Raman spectrum. The frequency of this main peak converges to the frequency of infinite bundles for bundles of 20 tubes. For larger tubes, the deformability of the tubes leads to the emergence of supplementary low-frequency modes. In Sec. IV B, we analyze the origin of these modes and the consequences of their specific signatures on the interpretation of experimental data.

(iv) Finally, we analyze the effect of the inhomogeneity of the bundle. We show that, if for small bundles (made of a few tubes) the individuality of the BM of each tubes is preserved, larger bundles (with more than ten tubes) have modes characteristic of the whole bundle and not of the individuals.

To obtain these results, we have used different models for the different type of interactions in the nanotube bundles. The intertube potential is modeled by the LJ potential in both microscopic and continuum forms. The intratube interaction have been considered within a valence force field (VFF) model for microscopic calculation on small systems (model II). The Raman intensity is then computed within the bondpolarization approximation. For larger systems, the tubes are considered as rigid cylinders that are only allowed to breathe. In that case, the Raman intensity is computed in an adapted interacting-dipoles scheme, which will be reported in the next section (model I).

The paper is organized as follow. The theoretical methods are introduced in Sec. II. The results on intertube modes are presented in Sec. III. Section IV A deals with isolated tubes and infinite bundles and, in Secs. IV B and IV C, we consider homogeneous and inhomogeneous finite bundles. Conclusions end the paper (Sec. V).

\section{MODEL FOR SIMULATION}

\section{A. Macroscopic model (model I)}

\section{Intertube interaction}

We first study vibrational modes by considering the nanotubes as continuous rigid cylinders characterized by a surface mass density $\sigma$ as in Ref. 12. The intertube van der Waals interactions are described by a $\mathrm{LJ}$ pair potential, $V_{c-c}=$ $-C_{6} / d^{6}+C_{12} / d^{12}$, where $d$ is the distance between two points on different tubes and $C_{6}$ and $C_{12}$ are constants fitted to reproduce the structural properties of graphite (Ref. 26). The potential $V_{c T}$ felt at a point of one of the tubes situated at a distance $a$ from the axis of the second tube of radius $R$ is given by $(a>R)$

$$
\begin{aligned}
V_{c T}(a)= & \frac{3}{4} \pi R \sigma\left\{\frac{C_{6}}{a^{5}} F\left[5 / 2,5 / 2,1,(R / a)^{2}\right]\right. \\
& \left.+\frac{21 C_{12}}{32 a^{11}} F\left[11 / 2,11 / 2,1,(R / a)^{2}\right]\right\},
\end{aligned}
$$

where $F$ is the hypergeometric function. ${ }^{27}$ The intertube interaction energy per unit length between two tubes, $V_{T T}$, is obtained by numerical integration of $V_{c T}$ over the surface of the first tube.

The equilibrium distance between tubes in a bundle are obtained by minimization of $V_{T T}$ with respect to the tubecenter coordinates. The dynamical matrix is calculated by numerical differentiation of $V_{T T}$ and the intertube vibrational modes are next deduced from the diagonalization of the dynamical matrix.

\section{Rigid breathing tubes}

In this model, we consider the tubes as continuous cylinders that are allowed to perform rigid breathing and displacement. We then have three degrees of freedom per tube, $\beta_{i}$ $(i=1, \ldots, 3 N), \beta_{i}$ stands for both the tube radii $\rho_{i}(i$ $=1, \ldots, N)$ and the tube centers coordinates in the $z=0$ plane, $\left(x_{i}, y_{i}\right)(i=1, \ldots, N)$ ( $N$ being the number of tubes in the bundle). The tube axis are assumed to be parallel to the $o z$ axis. The interaction potential reads

$$
V=V_{\text {intra }}\left(\rho_{i}\right)+V_{T T}\left(x_{i}, y_{i}, \rho_{i}\right),
$$

where $V_{T T}$ is described in Sec. II A. The dynamical matrix elements follow from $D_{i j}=\Phi_{i j} / \sqrt{m_{i} m_{j}}$ and 


$$
\Phi_{i j}=\frac{\partial^{2} V}{\partial \beta_{i} \partial \beta_{j}}=\frac{\partial^{2} V_{\text {intra }}}{\partial \beta_{i} \partial \beta_{j}}+\frac{\partial^{2} V_{T T}}{\partial \beta_{i} \partial \beta_{j}}
$$

and

$$
\frac{\partial^{2} V_{\text {intra }}}{\partial \rho_{i} \partial \rho_{j}}=k_{\text {intra }}^{i} \delta_{i j}
$$

For the sake of simplicity and reducing the computing time and in contrast to Ref. 12, we compute $k_{\text {intra }}^{i}$ from the empirical formula based on the VFF approach ${ }^{9}$ where the frequency $\omega_{(n, m)}$ of the BM has been found to depend on the tube radius and $\omega_{(n, m)}\left(\mathrm{cm}^{-1}\right)=1147 / r_{(n, m)}(\AA)$, where $r_{(n, m)}$ is the radius of the $(n, m)$ nanotube. $k_{\text {intra }}^{i}$ follows from $\omega=\sqrt{k_{\text {intra }}^{i} / m_{(n, m)}}$, where $m_{(n, m)}$ is the mass per unit length of a $(n, m)$ tube.

Since we consider three degrees of freedom per nanotube, a bundle made of $N$ nanotubes admits $3 N$ eigenmodes that are evaluated by the diagonalization of the dynamical matrix. The $3 N$ modes can be separated into $2 N$ intertube modes that appear when $\beta_{i}=x_{i}, y_{i}$ are considered (and $\beta_{i}=\rho_{i}$ being fixed) and $N$ radial-derived modes that appear when $\beta_{i}=\rho_{i}$ is considered ( $\beta_{i}=x_{i}, y_{i}$ being fixed). We checked numerically that such decoupling leads to an error smaller than $1 \%$ for the intertube modes and smaller than $0.1 \%$ for the radialderived modes. In the numerical application, we then decouple the $3 N \times 3 N$ system into one $2 N \times 2 N$ and one $N$ $\times N$ eigenvalue problem.

Next, we estimate the Raman intensity $I_{\mathbf{q}_{i}}$ for the $3 N$ modes characterized by the eigenvectors $\mathbf{q}_{i}, i=1, \ldots, 3 N$ by

$$
I_{\mathbf{q}_{i}} \sim\left|\left\langle\frac{\partial \boldsymbol{\alpha}}{\partial \mathbf{q}_{i}}\right\rangle\right|^{2} .
$$

The polarizability $\alpha$ of the ensemble of $N$ tubes is approximated from the polarizability per unit length of an ensemble of interacting linear density of dipoles, representing the ensemble of tubes.

Indeed, it was established ${ }^{28}$ that the static transverse polarizability per unit length of a nanotube scales like the square of its radius, independent of its chirality and that the static polarizability per angstrom of a $(10,10)$ tube is $32.71 \AA$. For the ensemble of interacting tubes, the total field, $\mathbf{E}_{i}$ at position $\mathbf{r}_{i}\left(\mathbf{x}_{i}, \mathbf{y}_{i}\right)$ is given by $\left(\mathbf{E}_{e x t}\right.$ denotes the external field)

$$
\mathbf{E}_{i}=\mathbf{E}_{e x t}+\sum_{j \neq i=1}^{N} \mathbf{T}^{\prime}\left(\mathbf{r}_{i}-\mathbf{r}_{j}\right) \mathbf{p}_{j}
$$

In Eq. (6), $\mathbf{p}_{i}=\boldsymbol{\alpha}_{i} \mathbf{E}_{i}$ and $\mathbf{T}^{\prime}\left(\mathbf{r}_{i}-\mathbf{r}_{j}\right)$ result from the integration over $z$ of the usual dipolar propagator $\mathbf{T}\left(\mathbf{r}_{i}-\mathbf{r}_{j}\right)$. Then $\left(r_{i j}^{2}=x_{i j}^{2}+y_{i j}^{2}, x_{i j}=x_{i}-x_{j}, y_{i j}=y_{i}-y_{j}\right)$

$$
T_{i j}^{x x^{\prime}}=\frac{2\left(x_{i j}^{2}-y_{i j}^{2}\right)}{r_{i j}^{2}},
$$

$$
T_{i j}^{x y}{ }^{\prime}=\frac{4 x_{i j} y_{i j}}{3 r_{i j}^{4}},
$$

$$
T_{i j}^{y y^{\prime}}=\frac{2\left(y_{i j}^{2}-x_{i j}^{2}\right)}{r_{i j}^{4}}
$$

for $i \neq j$, and for $i=j,\left(z_{k, l}=x, y\right)$

$$
T_{i j}^{z_{k} z_{l}^{\prime}}=0 .
$$

The $2 \times 2$ polarizability tensor of the bundle is then

$$
\boldsymbol{\alpha}=\sum_{i \neq j}\left[\left(\mathbf{1}_{2 N \otimes 2 N}-\boldsymbol{\Gamma} \mathbf{T}^{\prime}\right)^{-1} \boldsymbol{\Gamma}\right]_{i j} \mathbf{E}_{e x t},
$$

where $\boldsymbol{\Gamma}$ is a $2 N \times 2 N$ block-diagonal tensor built with the $N$ "atomic" polarizability $2 \times 2$ tensor $\alpha_{i}, \mathbf{T}^{\prime}$ is a $2 N \times 2 N$ tensor built with the $2 \times 2$ matrices $T_{i j}^{\prime}$ and $\mathbf{1}_{2 N \otimes 2 N}$ is the $2 N \times 2 N$ unit tensor. The sum runs over the $N^{2} 2 \times 2$ blocks of the tensor between [ ].

From Eq. (11), the variation of the static polarizability due to a given normal mode can be computed and the Raman intensity then follows from Eq. (5).

\section{B. Microscopic model valence force field and bond polarization (model II)}

Model II is a microscopic model where the carbon-carbon interactions are described by a VFF model with parameters for the following types of interaction: nearest-neighbor stretch, second nearest-neighbor stretch, in-plane bend, outof-plane bend, and twist. The VFF parameters are deduced from a fit of experimental data of graphite ${ }^{29}$ and have been used in several studies of the lattice dynamics of SWNTs. ${ }^{9,30,31}$ It has been verified that the chosen set of VFF parameters reproduce well the elastic properties of SWNTs. ${ }^{31}$

The interactions between carbon atoms belonging to different tubes are described by a LJ potential. Due to the lack of reliable structural data, it is assumed that for the equilibrium state all carbon bonds are equal to $1.42 \AA$, all bond angles are equal to each other and the tubes have circular cross sections. The latter assumption implies, in particular, that the effect of the intertube interactions on the equilibrium tube shape is ignored.

In the case of infinite crystals of $(n, m)$ tubes, the crystal is three dimensional with a unit cell containing $N_{t}$ carbon atoms $\left[N_{t}=2\left(n^{2}+m^{2}+n m\right) / d\right.$, where $d$ is equal to the highest common divisor $d^{\prime}$ of $n$ and $m$ if $n-m$ is not a multiple of 3 or $d$ is equal to $3 d^{\prime}$ if $n-m$ is a multiple of $3 d^{\prime}$ ]. For a finite bundle of $N$ tubes, the crystal is one-dimensional with $N N_{t}$ atoms in the unit cell. In the microscopic approach, the intertube interaction energy is minimized with respect to the intertube separation and the angle of simultaneous rotation of all tubes about their axes. The optimized intertube separations are roughly equal to $3.15 \AA$.

The dynamical matrix is constructed using force constants of the VFF type for the intratube interactions and force constants deduced from the LJ potential for the intertube inter- 


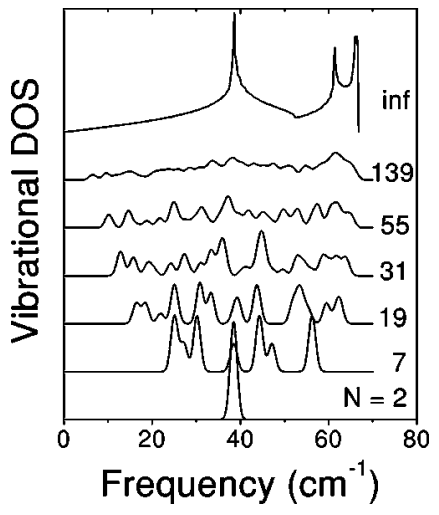

FIG. 1. Intertube vibrational density of states calculated in model I (rigid tubes) for bundles of $N$ tubes $(N$ $=2,7,19,31,55,139)$ with $r=6.8 \AA$. The top curve is for an infinite hexagonal array of nanotubes.

actions. The $\gamma$-point phonons for a given bundle are obtained by diagonalization of the $3 N_{t} \times 3 N_{t}$ or the $3 N N_{t} \times 3 N N_{t}$ dynamical matrix for the case of an infinite or finite bundle, respectively. Since this procedure is proportional to the cube of the size of the matrix, it can only be applied to tubes of certain types with small $N_{t}$ (e.g., armchair or zigzag) and for bundles with small $N$.

The Raman spectra are calculated within the bond polarizability (BP) model. ${ }^{33}$ In all cases the Raman intensity is averaged over all tube orientations in space.

\section{INTERTUBE MODES}

First, we deal with the pure intertube vibrational modes obtained from model I. For a finite number $N$ of tubes, the dynamical matrix was computed from the second derivative of the van der Waals energy with respect to the tube-center positions and, then, diagonalized to obtain the $2 \mathrm{~N}$ eigenmodes (including two translational modes and one rotational mode of zero frequency). In Fig. 1, the total intertube density of vibrational states (DOS) is presented for 2-139 tubes in a hexagonal bundle $\left(C_{6 v}\right.$ point group with one central tube for $N=6 i+1)$. The tube radii are chosen to be $6.8 \AA$, identical for all tubes. The $2 N-3$ finite eigen-frequencies are presented as spectra in Fig. 1 with a $3 \mathrm{~cm}^{-1}$ broadening used to the compare to the inelastic-neutron experiment or Brillouinscattering data when available. Amongst the vibrational modes, we found $i A_{1}$ and $2 i E_{2}$ Raman active modes and $2 i$ $E_{1}$ infrared active modes.

The frequencies of the $2 \mathrm{~N}-3$ optical modes were calculated and presented in Fig. 1 of Ref. 12. It is interesting to mention that for $N=2$ the only optical intertube mode is a symmetric one. For larger number of tubes, the mode structure becomes more complex but leads to a DOS for infinite array of NTs characterized by three singularities (top curve in Fig. 1). The analysis of the dispersion curves [Fig. 2(a)] gives $1435 \mathrm{~m} / \mathrm{s}\left(v_{L A}\right)$ and $831 \mathrm{~m} / \mathrm{s}\left(v_{T A}\right)$ as sound velocities at the $\Gamma$ point associated with the intertube branches.

We also report in Fig. 2(b) the total DOS of an infinite bundle of nanotubes with $r=3.39 \AA[(5,5)$ nanotube $]$ and $r=10.17 \AA[(15,15)$ nanotube $]$ together with the curve as-
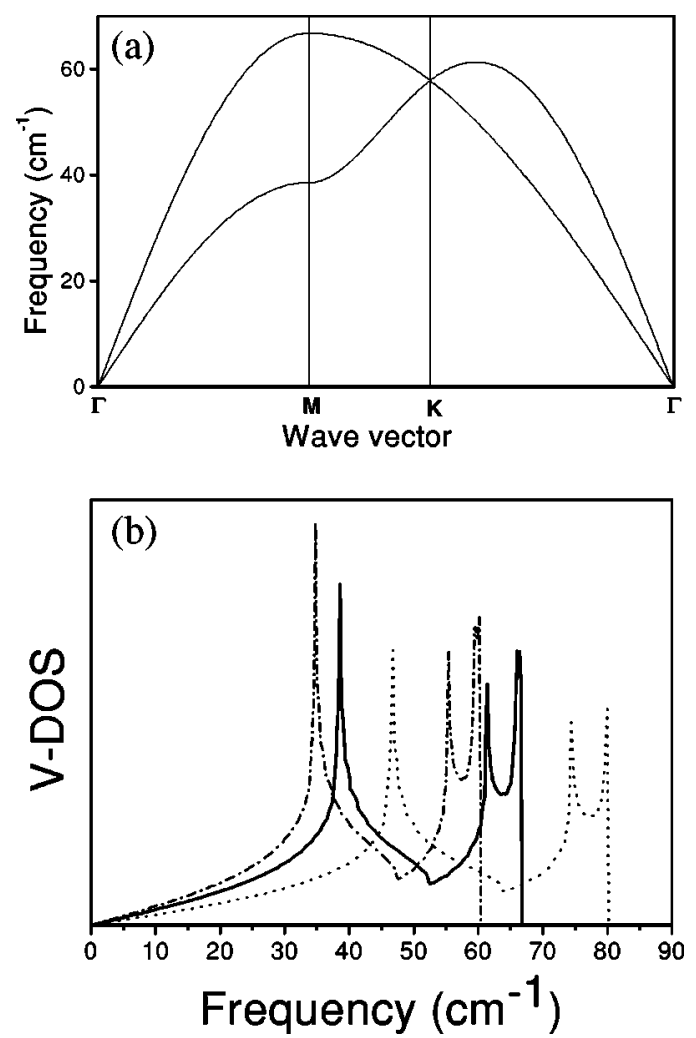

FIG. 2. (a) Dispersion curve of the intertube modes obtained within model I (rigid tubes). (b) Density of vibrational states for an infinite crystal of nanotubes for $r=6.8 \AA$ (solid line), $r=3.39 \AA$ (dotted line), and $r=10.15 \AA$ (dotted-dashed line).

sociated with $r=6.8 \AA$ (same as the top curve in Fig. 1). As a consequence of the potential described in Eq. (1), the vibrational frequencies increase with decreasing tube radii but the general shape of the DOS (and the dispersion curves) does not change. The sound velocities are $1024 \mathrm{~m} / \mathrm{s}$ and $591 \mathrm{~m} / \mathrm{s}$ for $r=3.39 \AA$ and $1814 \mathrm{~m} / \mathrm{s}$ and $1048 \mathrm{~m} / \mathrm{s}$ for $r=10.17 \AA$.

In the present calculation, we have considered a macroscopic continuum model (model I), i.e., the tubes are allowed to have a breathing motion but not to be deformed. We will see in the next section that this rigid model for the tube breaks down for tube larger than $(10,10)$ because the mixing of intratube and intertube modes. For example, the $E_{2 g}$ (squashing) mode of the isolated $(10,10)$ tube has a frequency in the range of frequencies of the intertube vibrations. A shift from $16 \mathrm{~cm}^{-1}$ to $41 \mathrm{~cm}^{-1}$ has been predicted when bundled. ${ }^{16}$ Another atomic motion that would couple with the intertube mode is the "twiston" mode. ${ }^{34}$ We note that the Lennard-Jones potential does not give a good description of the shear forces between graphene sheets and, then, would not give a satisfactory description of the modes that imply the related motion of the carbon atoms in nanotube bundles (as for the "twiston" mode). The detailed interpretation of the specific-heat curves as a function of the temperature ${ }^{24,25}$ would then imply further analysis of the intertube modes within a microscopic model and is beyond the scope of the present paper. 


\section{BREATHINGLIKE MODES IN BUNDLE}

\section{A. Infinite bundle}

Before dealing with a finite bundle of nanotubes, we describe here the predictions of the two models presented above for infinite periodic bundles of tubes. In model II, the low-frequency Raman spectrum of isolated tube is dominated by three active modes: a $E_{1 g}$, a $A_{1 g}$, and a $E_{2 g}$ mode. The $A_{1 g}$ phonon is the $\mathrm{BM}$ and is the most active mode. Its diameter dependence ${ }^{9,33}$ and the packing effect ${ }^{12,14,16}$ have already been reported. Recently, ${ }^{32}$ we have found a strong effect of the intertube interaction of the BM and, in particular, that, due to the lowering of the symmetry, two hybrid modes [BM(1) and $\mathrm{BM}(2)$, Fig. 3] with nonvanishing intensities appear when tubes bundle. For small nanotube diameter only $\mathrm{BM}(1)$ has an important intensity and this mode is associated with a nearly pure breathinglike eigenvector. This $\mathrm{BM}(1)$ mode is the mode that has been reported in previous studies. ${ }^{12,14,16}$ For tube radii larger than $0.7 \mathrm{~nm}$, the eigenvector of $\mathrm{BM}(1)$ shows a clear hexagonal deformation due to the intertube interactions. As a consequence the upshift of $\mathrm{BM}(1)$ becomes less important and, even for $r>1 \mathrm{~nm}$, the $\mathrm{BM}(1)$ mode has a frequency lower that the BM frequency of isolated tube. At the same time, the higher-frequency mode $\mathrm{BM}(2)$ becomes more and more intense. Further details are given in Ref. 32.

In Fig. 3(a), we display the diameter dependence of the pure radial modes of isolated tubes (open symbols) and the associated modes of bundles with a radial character $[\mathrm{BM}(1)$ and $\mathrm{BM}(2)]$ (solid symbols). The dotted line gives the frequency of the radial mode of a bundle of tubes when the van der Waals interaction is considered within model I (with a forced pure radial mode). We see that the forced radial mode gives representative results for $r<6 \AA$ only. This is an $a$ posteriori justification of the frozen-phonon approach for small tubes. ${ }^{12}$ In particular, the upward shift of $\mathrm{BM}(1)$ relative to the BM mode, $\Delta \omega$, is constant $\left(15 \mathrm{~cm}^{-1}\right)$ for $r$ $<6.5 \AA$. The same conclusion has been found in Ref. 12 $\left(\Delta \omega=20 \mathrm{~cm}^{-1}\right)$ and Ref. $7\left(\Delta \omega=12 \mathrm{~cm}^{-1}\right)$. For larger radii, the tube elasticity lowers the restoring force for the breathinglike modes and a sharp decrease of $\Delta \omega$ is observed. We note that the radii range of constant $\Delta \omega$ corresponds to the radii range for which model I (rigid tubes) remains valid.

In a recent review paper, ${ }^{7}$ the breathinglike modes in infinite bundles have been reported for tube radii below $9.5 \AA$. The authors use a tight-binding model for the intratube interactions and a LJ potential for the intertube interactions. Two breathinglike modes (called $\beta$ and $\gamma$ ) were also predicted. It was found that the frequencies of the most intense mode converge with increasing radii to the frequency of BM of isolated tubes but with no crossing below $9.5 \AA$. However, extrapolating their result, such a crossing is likely to take place close to $9.5 \AA$. Furthermore, in Ref. 7 , the relative intensities of the breathinglike modes were calculated by projection of their eigenvectors onto that of BM. The results for relative intensities are in fair agreement with the prediction of model II (Ref. 32).

The frequency dependence of the BM is commonly described by the formula $\omega\left(\mathrm{cm}^{-1}\right)=C / r^{\alpha}(\AA)$. In the case of
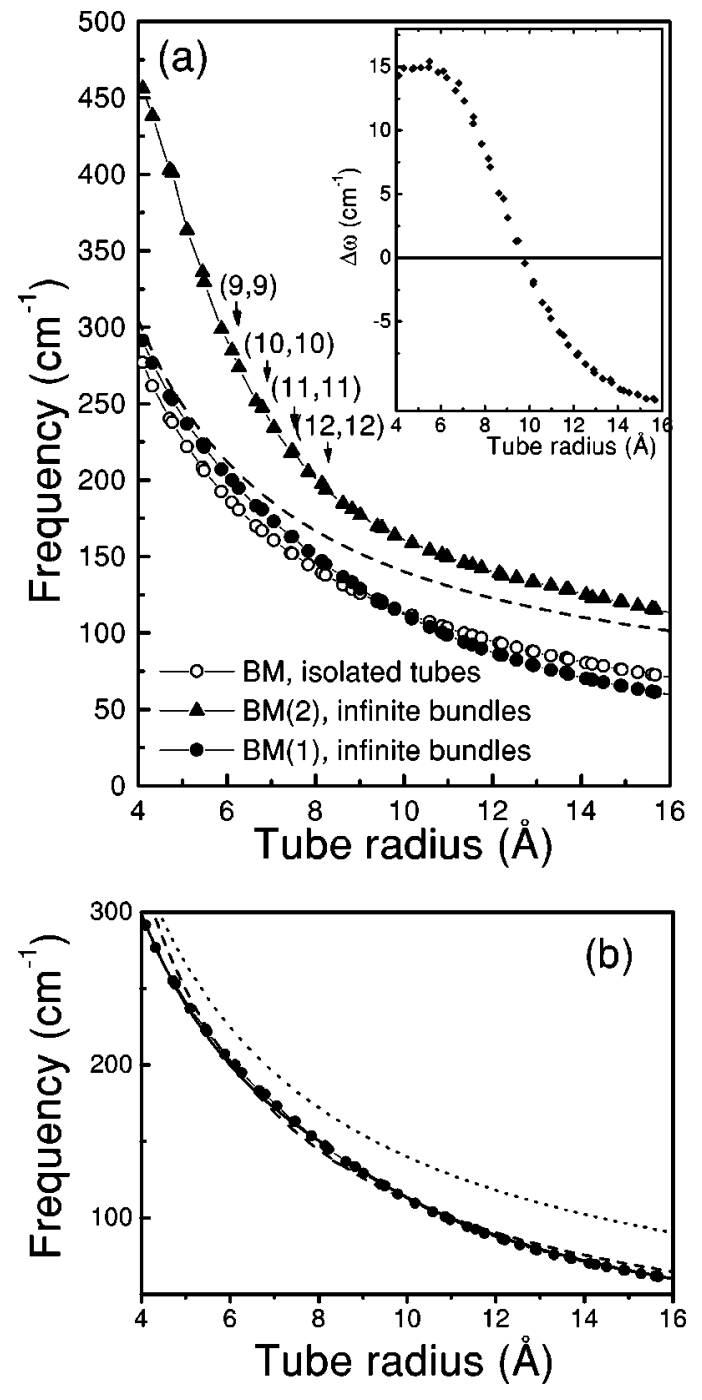

FIG. 3. (a) Summary of the calculated breathing-mode frequencies of isolated tubes and infinite arrays of nanotubes as a function of the tube radius. Results from model II: isolated nanotubes (BM) (open circles), bundles of nanotubes $\mathrm{BM}(1) / \mathrm{BM}(2)$ (Solid circles/ squares). The dotted line is the result of the model I (rigid tubes) for infinite bundles of tubes. Inset: frequency shift of the main Raman peak when nanotubes are bundled $\Delta \omega=\omega(B M(1))-\omega(B M)$. (b) Illustration of the fitting procedure. Solid circles: simulated frequencies for BM (1). Solid curve: Separated fit for $4.5<r(\AA)$ $<8.5 \AA$ and $8.5<r(\AA)<17 \AA$ (see text). Dashed line: Best fit for $4.5<r(\AA)<17 \AA$ (see text). Dotted line: Fitting curve of Rols et al. (Ref. 35).

isolated tubes, model II has given $C=1147 \AA / \mathrm{cm}^{-1}$ and $\alpha=1.00181$ (the least-square fit gives $\chi^{2}=0.04$ ) (Ref. 9). For the $\mathrm{BM}(1)$ and $\mathrm{BM}(2)$ of a bundle of nanotubes, the predictions of model II did not allow a satisfactory fitting: for $\mathrm{BM}(1)$, we obtained $C=1613 \AA \mathrm{Am}^{-1}$ and $\alpha=1.16$ $\left(\chi^{2}=21\right)$ [see Fig. 3(b), dashed line] but the fitted curve overestimates the data for $r<5 \AA$ and $r>12 \AA$ and underestimates them for $7 \AA<r<9 \AA$. When two separated radii ranges are considered, a better fit is found: $C$ $=1179 \AA / \mathrm{cm}^{-1}$ and $\alpha=0.99$ for $4.5<r(\AA)<8.5 \AA\left(\chi^{2}\right.$ $=2.45)$ and an excellent fit for $C=2536 \AA / \mathrm{cm}^{-1}$ and $\alpha$ 


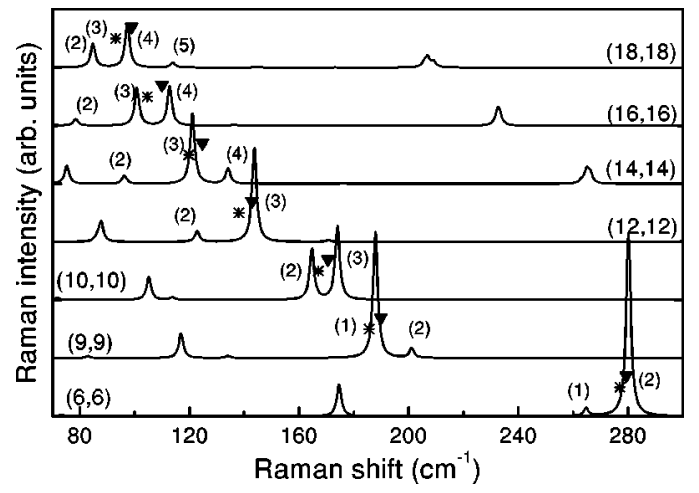

FIG. 4. Calculated Raman spectra of dimer of $(n, n)$ tubes ( $n$ $=6,9,10,12,14,16,18)$ in the energy range of the breathing modes obtained by model II. The stars give the position of the BM of isolated tubes. The down triangles are the position of the Raman active mode within model I. The labels (1)-(5) refer to the different modes with different symmetries (see text).

$=1.35$ for $8.5<r(\AA)<17 \AA\left(\chi^{2}=0.08\right)$ (see Fig. 3(b), solid line]. Rols et al. found ${ }^{35}$ a value of $C$ $=1190 \AA / \mathrm{cm}^{-1}$ and $\alpha=0.93$ for a bundle of tubes with radii between $3.5 \AA$ and $12.5 \AA$ ) [see Fig. 3(b), dotted line].

For $\operatorname{BM}(2)$, the fit with one single power law was also unsatisfactory. Dividing the radii range into two parts yields $C=3218 \AA \mathrm{Am}^{-1}$ and $\alpha=1.33$ for $4.5<r(\AA)<8.5 \AA\left(\chi^{2}\right.$ $=2.52)$ and $C=988 \AA / \mathrm{cm}^{-1}$ and $\alpha=0.78$ for $8.5<r$ $<17 \AA\left(\chi^{2}=2.03\right)$. These numbers have no physical meaning but come out from a fitting procedure and could be used when comparing these results with experimental data. However, the fact that we found two regimes with a crossover at $8.5 \AA$ has to be related to the deformation of the tube that becomes important above this value.

\section{B. Finite homogeneous bundles}

We now turn to the study of finite bundles of identical tubes called "homogeneous bundles." Figure 4 presents the calculated Raman intensity of a dimer of armchair $(n, n)$ tubes $(n=6,9,10,12,14,16$, and 18) obtained by model II. The major peaks are denoted by (1)-(5). In the same figure, the stars indicate the position of the BM modes of isolated tubes and the solid down triangles indicate the frequencies of the in-phase breathing mode in model I. Here, we recall that, in the model I, the number of modes of a bundle made of $N$ tubes is $N$. For a two-tube bundle, the modes are characterized by a pure in-phase and a pure out-of-phase breathing. The later mode has no Raman activity and lies very close to the energy of the isolated-tube mode because of the very small tube-tube interaction for such atomic motion. The inphase mode is upshifted as described in Fig. 4.

The atomic displacements for two high-intensity modes for some of the dimers are shown in Fig. 5. For the small $(6,6)$ tubes, the most intense mode of the dimer $\left[280 \mathrm{~cm}^{-1}\right.$, mode (2)] is characterized by an almost radial atomic displacement for each tubes. As a consequence, the two models give similar results $\left(279.4 \mathrm{~cm}^{-1}\right.$ in model I and $280 \mathrm{~cm}^{-1}$ in model II). For the other mode with frequency of
$(6,6)$

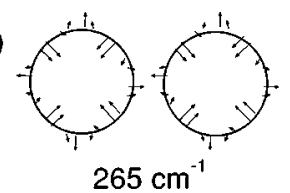

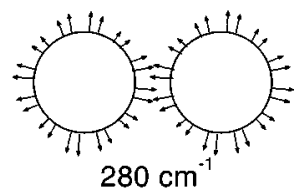

$(10,10)$
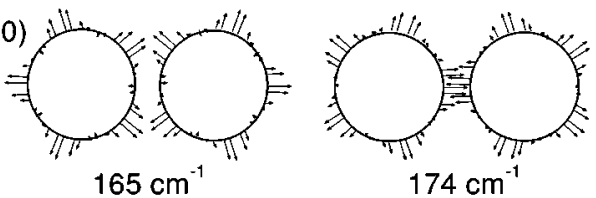

$(16,16)$

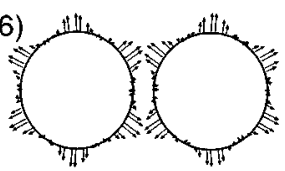

$101 \mathrm{~cm}^{-1}$

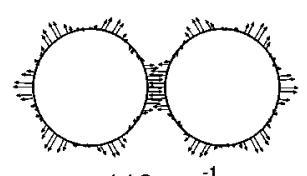

$113 \mathrm{~cm}^{-1}$

FIG. 5. Atomic-displacement patterns for the two major breathinglike modes of dimers of $(6,6),(10,10)$, and $(16,16)$ nanotubes.

$265 \mathrm{~cm}^{-1}$ [mode (1)], the vibration pattern is close to that of a $E_{4}$ mode of isolated $(6,6)$ tubes.

For larger tubes, the fact that the tubes are easier to deform leads to a more important hybridization between the $\mathrm{BM}$ and the $E_{n}$ modes of isolated tubes. For dimers of $(10,10)$ tubes, the atomic displacement for modes (3) and (4) (Fig. 5) are found to be nearly the same, apart from a rotation about the axis of the tube (Fig. 6). This reveals a strong mixing between the $\mathrm{BM}$ and the $E_{5}$ modes of the isolated tubes when they are brought together to form a dimer.

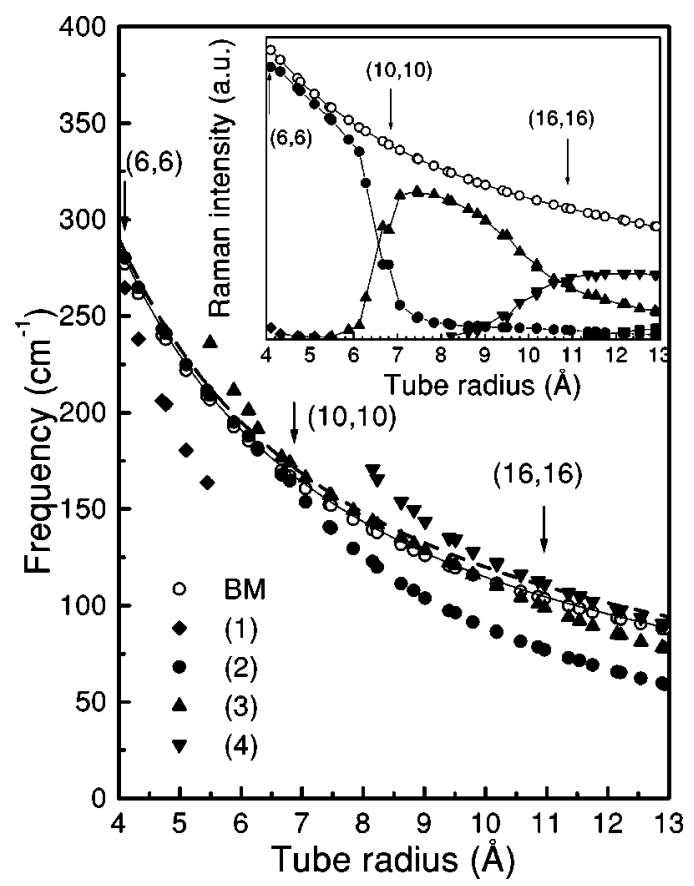

FIG. 6. Summary of the frequencies of the main breathinglike modes of isolated tubes and dimer of armchair and zigzag tubes obtained in model II. The labels (1),(2), ...(5) refer to Raman peak of Fig. 4. The open circles are for isolated tubes. The dashed line gives the position of the in-phase breathing mode within model I. The inset presents the intensities of the most intense modes. 


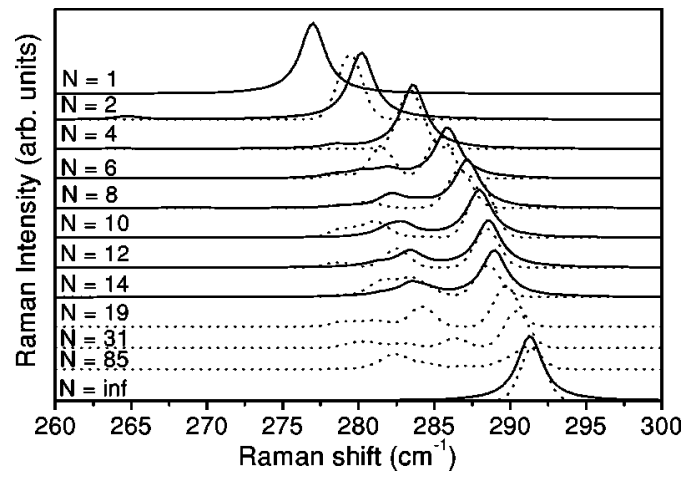

FIG. 7. Low-frequency Raman spectra of finite size $(6,6)$ bundles in model I (dotted line) and in microscopic model II (solid lines) as a function of the number of tubes in the bundle.

Dimers of tubes $(16,16)$ show two intense modes [(3) and (4)] with very similar intensities (Fig. 5) that originate from a coupling between the BM and the $E 6$ modes.

We display the main information on radius dependence of Raman spectra of dimer of all armchair and zigzag tubes with $4 \AA<R<13 \AA$ in Fig. 6. The main panel gives the frequencies of all the modes with notable intensities. The open circles are for isolated tubes and are shown for comparison. The dashed line gives the positions of the in-phase breathing mode within macroscopic model I. The inset resumes the intensities of the selected modes obtained by the bond-polarization approach. It is worth emphasizing that for radii close to $7 \AA$ (that are most often met in experimental samples), there exists two breathinglike modes of comparable Raman intensities with a frequency separation of $10 \mathrm{~cm}^{-1}$. This separation is large enough to be observed as separate peaks in experimental spectra.

The transition from isolated tubes or dimers of tubes to the case of infinite bundles is shown in Fig. 7 for $(6,6)$-tubebased bundles and in Fig. 8 for $(10,10)$-tube-based bundles for both macroscopic model I (dotted curve) and microscopic model II (solid curve). The rigid character of $(6,6)$ tubes leads to a similar behavior of the in-phase (high-energy) breathing mode computed in both approaches. We note that the convergence to the infinite-tube case is already very good at $N=20$ and almost achieved at $N=31$.

The $(10,10)$-tube case is rather different. First, as already mentioned, the infinite bundle $\mathrm{BM}(1)$ is not at the same en-

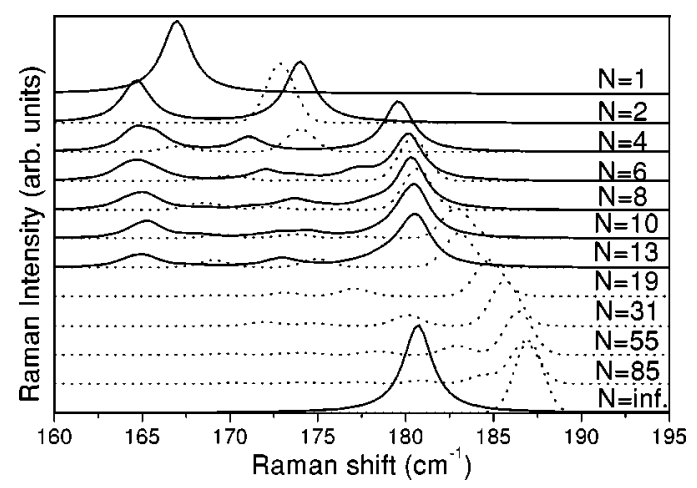

FIG. 8. Same is Fig. 7 but for bundle of $N$ tubes $(10,10)$.

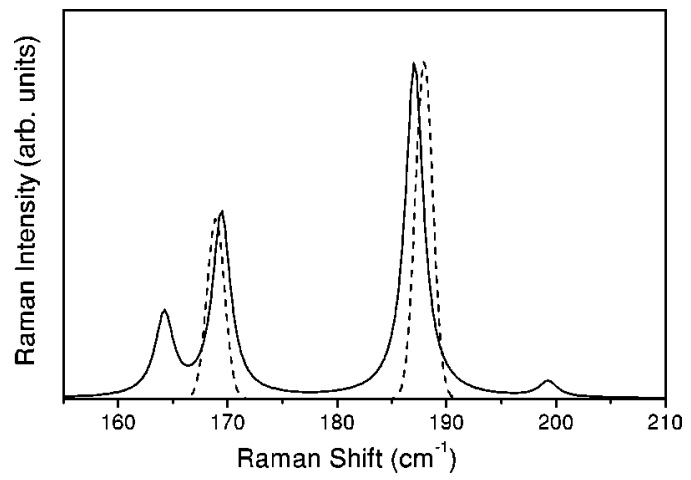

FIG. 9. Calculated Raman spectra of a dimer of $(9,9)-(10,10)$ tubes. The model II results are represented by solid lines. Dotted line is obtained within model I.

ergy when computed in different approaches. Due to the deformability of the tubes, the intertube interactions are effectively damped at relatively short distances. Therefore, the convergence of the breathinglike mode frequency is faster within model II. Note that mode (2) has a nearly constant frequency and is observable for bundle up to 12 tubes. Therefore this mode is not only specific to a dimer of tubes but also to finite bundles in this diameter range.

We have also studied larger tube bundles. In particular, we checked that the $\mathrm{BM}(2)$ mode observed for infinite bundles (Sec. IV A) is negligible for the $N<10$ bundle and therefore it is a specific signature of bigger nanotube crystals.

\section{Inhomogeneous bundles}

Previously, we have studied the vibrational structure of bundle of identical tubes. But the present production techniques could lead to the formation of bundles constituted by tubes of similar but not identical diameters. We analyze in this section the role of tube-radii inhomogeneity in the bundle.

We start with a dimer made of a $(9,9)$ and a $(10,10)$ tube. Figure 9 shows the obtained spectra within model I (dotted curve) and model II (solid curve). From the analysis of the eigenvectors, we concluded that the lower-frequency pair of the peaks is mainly related to a vibration of the $(10,10)$ tube and that the higher-frequency pair of peaks can be associated to a vibration of the $(9,9)$ tube. Model I (rigid tubes) gives only two modes but catches the essential features, i.e., the separation between the two (groups of) modes. The major peaks in both bands are very close to the vibrational frequency of the isolated tube $\left[188 \mathrm{~cm}^{-1}\right.$ for $(9,9)$ tube and $169 \mathrm{~cm}^{-1}$ for $(10,10)$ tubes]. This demonstrates that in such a case (small bundle made of tubes with rather different vibrational frequencies when isolated) the experimental spectra would allow one to evidence the individual component of the bundle even if the modes are split due to the lower symmetry of the environment.

Due to computational limitations, the breathinglike modes of larger inhomogeneous bundles are only studied here within model I. We have considered bundles consisting of a central tube with layers of six tubes around it. All tubes have the same radii $\left(r_{A}=6.8 \AA\right.$, type $\left.A\right)$ except one (type $\left.B\right)$ that 


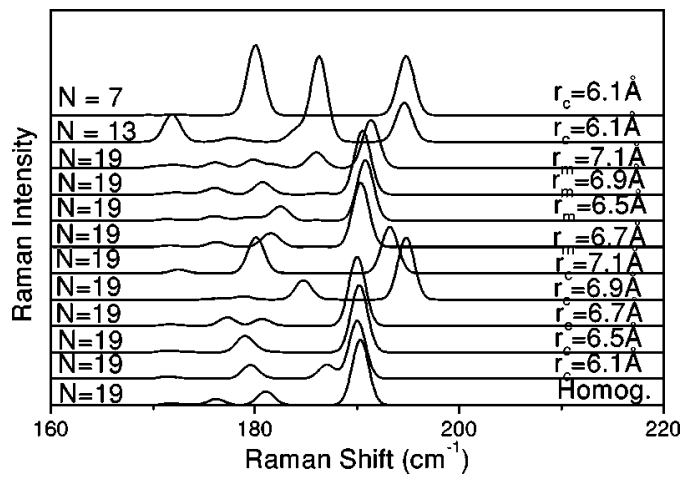

FIG. 10. Raman spectra of inhomogeneous finite bundles within the model I. $N$ is the number of tube in the bundle (a central nanotube surrounded by shells of six tubes is assumed). $r_{c}\left(r_{m}\right)$ are the diameter of central (off-center) type- $B$ nanotubes. Type- $A$ nanotubes have a radius $r=6.8 \AA$.

can occupy a central position (radius $r_{c}$ ) or an off-central position (radius $r_{m}$ ). In Fig. 10, we present the calculated Raman spectra for different number of tubes $N$ and different radii and positions of the type- $B$ tube.

For $N=7$ tube bundle and $r_{c}=6.1 \AA$, two modes (194.8 $\mathrm{cm}^{-1}$ and $180.0 \mathrm{~cm}^{-1}$ ) have an observable Raman activity (Fig. 10). The mode at $194.8 \mathrm{~cm}^{-1}$ is characterized by the central type- $B\left(r_{c}=6.1 \AA\right)$ tube breathing and the mode at $180.0 \mathrm{~cm}^{-1}$ is characterized by a predominant participation of the six external tubes (together with a counterphase vibration of the central tube $B$ ). In other words, in such cases the eigenvectors are dominated by the excitation of one type of tubes and this explains the two distinct peaks with similar intensities. Note also that the isolated $r$ $=6.1 \AA$ tube has a BM at $188 \mathrm{~cm}^{-1}$ and the isolated $r$ $=6.8 \AA$ tube has a BM at $168 \mathrm{~cm}^{-1}$.

For $N=13$ tube bundles and $r_{c}=6.1 \AA$, the four most intense modes are at $194.6 \mathrm{~cm}^{-1}$ [mode (1)], $186.2 \mathrm{~cm}^{-1}$ [mode (2)], $184 \mathrm{~cm}^{-1}[\operatorname{mode}(3)]$, and $171 \mathrm{~cm}^{-1}[\operatorname{mode}$ (4)]. Mode (1) involves a breathing of the central tube $B$ with small in-phase motion of the first surrounding layer of six tubes. Modes (2) and (3) form a single peak and involve mainly a breathing of the first and second layers (type- $A$ tubes) and a counterphase breathing of the central small tube $B$. Mode (4) is characterized by an counterphase breathing of the first and second layers and a nonbreathing of the central $B$ tube.

For a $N=19$ bundle tubes $\left(r_{A}=6.8 \AA\right.$ and $\left.r_{c}=6.1 \AA\right)$, we obtained a spectrum very similar to that of a homogeneous bundle (Fig. 10). The high-frequency mode at $190.0 \mathrm{~cm}^{-1}$ corresponds to a breathinglike mode with an in-phase vibration of tube $A$ and a counterphase vibration of type $B$. And there is no clear spectral signature of the two tube types. We have also performed simulations for various values of $r_{c}$ and $r_{m}$ (Fig. 10). The general conclusion is that for $r_{c}<r_{A}$ the main line is not influenced significantly by the central type- $B$ nanotube. For $r_{c}>r_{A}$, the main line is slightly shifted upwards and the corresponding eigenvector represents an in-phase motion of both $A$ and $B$ tubes. In the case of an off-center $B$ tube, the symmetry of the bundle is lowered and all the 19 breathinglike modes are active. However,

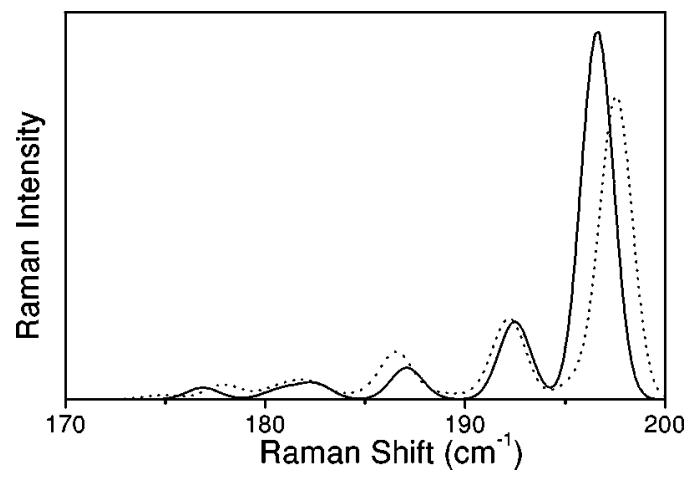

FIG. 11. Raman spectra of 55 tubes bundle within the rigid model (model I). The solid line is for an homogeneous bundle with $r=6.8 \AA$. The dotted line is for a 55-tube bundle with tube radii in the interval $6.7 \AA<r<6.8 \AA$.

the main line remains associated with the mode with inphase breathing of all the tubes.

Calculation of Raman spectra of larger bundles were also performed. In Fig. 11, we present the spectrum for a bundle of 55 tubes of radii $r=6.8 \AA$ in comparison with the spectrum with introduced "disorder" in the radii, i.e., we have chosen tubes with ten different radii within $0.1 \AA$ around $r$ $=6.8 \AA$. The procedure for the construction of the bundle is the same as described in Ref. 2 where a bundle of 55 tubes with random chirality was chosen to simulate the electron diffraction from an individual bundle of tubes. The main effect of the disorder is a shift and a small broadening of the main radial-mode line. It is worth noting here that there is no more symmetry in the bundle and consequently all the 55 modes are active. It is important also to note that BM frequency spreading of isolated tubes with radii ranging from $6.7 \AA$ to $6.9 \AA$ is larger than $5 \mathrm{~cm}^{-1}$. The broadening we obtained in the present simulation is much smaller and, then, we conclude that the Raman spectra of such "disordered" bundles cannot be considered as a superposition of the signatures of the 55 individual tubes. We think that such a conclusion will remain valid in a microscopic model (model II). Moreover, the possibility of the deformation of the section of the tubes will accentuate the coupling between tubes of different radii and chiralities.

\section{DISCUSSION}

The only experimental evidence of a change of the BM frequency when nanotubes are bundled is the small shift (4\%) obtained by Kataura et al. ${ }^{17}$ when comparing Raman spectra of Br-doped and -undoped nanotube samples. If confirmed, this would imply a more important deformability of the tube than predicted here or a less important van der Waals interaction.

However, the most direct verification of the present calculations would be the observation of the additional breathinglike $\mathrm{BM}(2)$ mode for large bundles or the observation of the hybrid breathinglike modes in the case of small bundles. Since most of the production methods give bundles made of a large number of tubes, the observation of $\operatorname{BM}(2)$ is expected. 
The most direct experimental evidence of the $\mathrm{BM}(2)$ mode is found in the work of Tan et al. ${ }^{36}$ where two lines are observed that correspond almost perfectly to the predicted $[\mathrm{BM}(1), \mathrm{BM}(2)]$ frequencies: $(157,215) \mathrm{cm}^{-1}$ [Fig. 1(a) of Ref. 36] and $(189,254) \mathrm{cm}^{-1}$ [Fig. 1(b) of Ref. 36]. In this work, we found (Fig. 3) $(159,214) \mathrm{cm}^{-1}$ for $r=7.6 \AA$ and $(187,260) \mathrm{cm}^{-1}$ for $r=6.5 \quad \AA$ for $[\mathrm{BM}(1), \mathrm{BM}(2)]$. A critical analysis of other published data has been presented in Ref. 32. However, we point out that our result allows an explanation in terms of resonant Raman data that have been previously analyzed in terms of nonresonant lines.

A very recent experimental study has been reported by Azoulay et $a l .{ }^{22}$ They obtained low-frequency-range spectra of a single object deposited on silver substrate in a backscattering geometry with a confocal microscope. Their spectra show two intense peaks at $205 \mathrm{~cm}^{-1}$ and $332 \mathrm{~cm}^{-1}$ when both incident and scattered light are parallel to the tube axis (VV polarization) and one mode at $147 \mathrm{~cm}^{-1}$ when scattered light in perpendicular to the tube axis ( $\mathrm{VH}$ polarization). The larger separation of the peaks excludes the presence of small bundles for which a specific signature has been predicted. Since both lines at $205 \mathrm{~cm}^{-1}$ and $332 \mathrm{~cm}^{-1}$ are observed in the same polarization and because $\mathrm{BM}(1)$ and $\mathrm{BM}(2)$ have similar eigenvectors, we assigned these two lines to the $\mathrm{BM}(1)$ and $\mathrm{BM}(2)$ modes that are predicted at $207 \mathrm{~cm}^{-1}$ and $299 \mathrm{~cm}^{-1}$ for $r=5.9 \AA$. The numerical correspondence is not so good and the intensity of the $\mathrm{BM}(2)$ mode for such a small tube is predicted to be very small. But the alternative explanation proposed in Ref. 22 involves the $E_{2}$ mode, predicted at $415 \mathrm{~cm}^{-1}$. The influence of interaction with the substrate could be part of the explanation for the present discrepancies.

Duesberg et al. have reported spectra from individual clusters in Refs. 18 (SERS on metal substrate) and 19 (glass substrate). Scanning electron microscopy and atomic force microscopy images allowed them to postulate that they studied individual SWNT or small clusters (3-5 nanotubes). In Ref. 18, they observed bands at 166, 191 , and $196 \mathrm{~cm}^{-1}$ (Fig. 2 of Ref. 18) and $165 \mathrm{~cm}^{-1}$ (Fig. 3 of Ref. 18). From our simulation on finite bundles (Figs. 4 and 5), we expected double peaks for breathinglike modes around $160 \mathrm{~cm}^{-1}(r$ $=7 \AA$ ) and one dominant peak for breathinglike modes around $190 \mathrm{~cm}^{-1}(r=6 \AA)$. So, without additional experimental results (better local-probe characterization or other laser wavelengths for the Raman experiment), it is difficult to conclude on this experiment. The authors of Ref. 19 observed one single Raman line around $190 \mathrm{~cm}^{-1}$, which is what is expected for isolated tubes with $r=5.7 \AA$. Note, however, that the width of the experimental Raman lines are very large and that a multipeak structure could give a refined measurement.

In Raman measurements of nanotube samples under pressure, ${ }^{14,37}$ the deformation (faceting) is expected to play a major role. However, no evidence of the $\mathrm{BM}(2)$ mode has been reported. We also note that an hexagonal deformation of large tubes has been observed without any applied pressure. ${ }^{38}$

Concluding this section, we emphasize that the appearance of the $\operatorname{BM}(2)$ mode in big bundles is supported by several experimental data and, moreover, could circumvent the analysis of the Raman spectra in terms of off-resonant excitations. For what concerns individual small bundles, further experimental and adapted theoretical work is needed to achieve a satisfactory correspondence. Experimental work with different laser frequencies would certainly give information about the number of tubes in the probed bundles and the analysis of resonant Raman spectra of a bundle of tubes remains a theoretical challenge.

\section{CONCLUSION}

In conclusion, we have presented detailed Raman-spectra simulations for finite and infinite bundles of SWNT in the frequency range associated with the breathing mode of isolated tubes (100-300 $\left.\mathrm{cm}^{-1}\right)$. We have emphasized the consequences of the intertube coupling on these modes. For infinite bundles, we have predicted the presence of a second mode of breathing character [called BM(2)] for $r>7 \AA$ as a consequence of the hybridization between the $\mathrm{BM}$ and a $E_{n}$ mode of isolated tubes (see also Ref. 32). The frequency of $\mathrm{BM}(1)$ has an upward shift of $15 \mathrm{~cm}^{-1}$ for small radii, diminishes to zero for $r=10 \AA$, and has a downward shift for larger radii.

With the tube dimers, we analyzed the consequences of the coupling of the $\mathrm{BM}$ mode with the $\mathrm{E}_{n}(n>2)$ mode of isolated tubes. This leads to modes with mixed character and to the observation of two peaks in the low-frequency Raman spectra for tube with radius around $6.8 \AA$ such as experimentally produced. We predicted that bundles constituted of larger number of tubes (up to ten) are still characterized by this specific signature.

Finally, we considered a simulation of inhomogeneous bundles (bundles made of tubes with nonuniform radii). We showed that large bundles made of a distribution of tube radii with small variance reacting like a system of oscillators and global modes are observed. Signature of individual tubes is only possible for small bundles with very different tube radii. As it has been shown experimentally ${ }^{2,39}$ that individual bundles are made of tubes with very similar radii, the analysis of breathing modes of nanotube crystals are not going to be able to resolve individual tube features and would exhibit the response of the entire nanocrystal. Then, each individual bundle has to be analyzed as a specific case.

\section{ACKNOWLEDGMENTS}

L.H. thanks Ph. Lambin for stimulating discussions. L.H. was supported by the Belgian National Fund (FNRS). V.N.P. acknowledges the financial support by Grant No. G.0347.97 of the Flemish Science Foundation, by the Concerted Action of the University of Antwerpen GOA-BOF-UA, and by the NATO Research program for Belgium. This work was also partly funded by the Belgian Interuniversity Research Project on Reduced Dimensionality Systems (PAI-UAP P4-10). A.R. acknowledges support from the sabbatical program Salvador de Madariaga of the Spanish MEC (PR2000-0335) and the Ecole Polytechnique, by the European Community through its RTN network Contract Nos. HPRN-CT-2000-00128 (COMELCAN), HPRN-CT-2000-00167 (NANOPHASE) and the RTD-FET program (SATUNET). 
*Permanent address: Faculty of Physics, University of Sofia, BG1164 Sofia, Bulgaria.

${ }^{1}$ C. Journet and P. Bernier, Appl. Phys. A: Mater. Sci. Process. 67, 1 (1998).

${ }^{2}$ L. Henrard, A. Loiseau, C. Journet, and P. Bernier, Eur. Phys. J. 13, 661 (2000).

${ }^{3}$ S. Rols, R. Almairac, L. Henrard, E. Anglaret, and J.L. Sauvajol, Eur. Phys. J. A 10, 263 (1999).

${ }^{4}$ L. Venema, V. Meunier, P. Lambin, and C. Dekker, Phys. Rev. B 61, 2991 (2000).

${ }^{5}$ A. Rubio, D. Sanchez-Portal, E. Artacho, P. Ordejón, and J.M. Soler, Phys. Rev. Lett. 82, 3520 (1999).

${ }^{6}$ P. Eklund, J. Holden, and R. Jishi, Carbon 33, 959 (1995).

${ }^{7}$ M. Dresselhaus and P. Eklund, Adv. Phys. 49, 705 (2000).

${ }^{8}$ R. Jishi, L. Venkataram, M. Dresselhaus, and G. Dresselhaus, Chem. Phys. Lett. 209, 77 (1993).

${ }^{9}$ V.N. Popov, V.E. VanDoren, and M. Balkanski, Phys. Rev. B 59, 8355 (1999).

${ }^{10}$ J. Kürti, G. Kresse, and H. Kuzmany, Phys. Rev. B 58, R8869 (1998).

${ }^{11}$ D. Sánchez-Portal, E. Artacho, J.M. Soler, A. Rubio, and P. Ordejón, Phys. Rev. B 59, 12678 (1999).

${ }^{12}$ L. Henrard, E. Hernandez, P. Bernier, and A. Rubio, Phys. Rev. B 60, R8521 (1999).

${ }^{13}$ M. Milnera, J. Kurti, M. Hulman, and H. Kuzmany, Phys. Rev. Lett. 84, 1324 (2000).

${ }^{14}$ U.D. Venkateswaran, A.M. Rao, E. Richter, M. Menon, A. Rinzler, R.E. Smalley, and P.C. Eklund, Phys. Rev. B 59, 10928 (1999).

${ }^{15}$ N. Hamada, S.I. Sawada, and A. Oshiyama, Phys. Rev. Lett. 68, 1579 (1992).

${ }^{16}$ D. Kahn and J.P. Lu, Phys. Rev. B 60, 6535 (1999).

${ }^{17}$ H. Kataura, Y. Maniwa, S. Masubushi, S. Kazama, X. Zhao, Y. Ando, Y. Ohtsuka, S. Suzuki, Y. Achiba, and R. Saito, in Electronic Properties of Novel Materials - Molecular Nanostructures, XIV International Winterschool/Euroconference, Kirchberg, edited by H. Kuzmany et al. (AIP, New York, 2000), p. 262.

${ }^{18}$ G.S. Duesberg, W.J. Blau, H.J. Byrne, J. Muster, M. Burghard, and S. Roth, Chem. Phys. Lett. 310, 8 (1999).

${ }^{19}$ G.S. Duesberg, I. Loa, M. Burghard, K. Syassen, and S. Roth, Phys. Rev. Lett. 85, 5436 (2000).
${ }^{20}$ P. Corio, S.D.M. Brown, A. Marucci, M.A. Pimenta, K. Kneipp, G. Dresselhaus, and M.S. Dresselhaus, Phys. Rev. B 61, 13202 (2000).

${ }^{21}$ J. Azoulay, A. Debarre, A. Richard, P. Tchnio, S. Bandow, and S. Iijima, Chem. Phys. Lett. 331, 347 (2000).

22 J. Azoulay, A. Debarre, A. Richard, P. Tchnio, S. Bandow, and S. Iijima, Eur. Phys. J. (to be published).

${ }^{23}$ K. Kneipp, H. Kneipp, P. Corio, S.D.M. Brown, K. Shafer, J. Motz, L.T. Perelman, E.B. Hanlon, A. Marucci, G. Dresselhaus, and M.S. Dresselhaus, Phys. Rev. Lett. 84, 3470 (2000).

${ }^{24}$ A. Mizel, L.X. Benedict, M.L. Cohen, S.G. Louie, A. Zettl, N.K. Budraa, and W.P. Beyermann, Phys. Rev. B 60, 3264 (1999).

${ }^{25}$ J. Hone, B. Batlogg, Z. Benes, A.T. Johnson, and J.E. Fisher, Science 289, 1730 (2000).

${ }^{26} \mathrm{~J}$. Israevlachvili, Intermolecular and Surfaces Forces (Academic Press, San Diego, 1992).

${ }^{27}$ Handbook of Mathematical Functions, edited by M. Abramowitz and I. Stegun (Dover, New York, 1968).

${ }^{28}$ L.X. Benedict, S.G. Louie, and M.L. Cohen, Phys. Rev. B 52, 8541 (1995).

${ }^{29}$ T. Aizawa, R. Souda, S. Otani, Y. Ishizawa, and C. Oshima, Phys. Rev. B 42, 11469 (1990); ibid. 43, 12060 (1991).

${ }^{30}$ V.N. Popov, V.E. VanDoren, and M. Balkanski, Phys. Rev. B 61, 3078 (2000).

${ }^{31}$ V.N. Popov, V.E. VanDoren, and M. Balkanski, Solid State Commun. 114, 395 (2000).

${ }^{32}$ V.N. Popov and L. Henrard, Phys. Rev. B 63, 233407 (2001).

${ }^{33}$ R. Saito, G. Dresselhaus, and M. Dresselhaus, Physical Properties of Carbon Nanotubes (Imperial College Press, London, 1998).

${ }^{34}$ Y.K. Kwon and D. Tomanek, Phys. Rev. Lett. 84, 1483 (2000).

${ }^{35}$ S. Rols, A. Righi, L. Alvarez, E. Anglaret, C. Journet, P. Bernier, J.L. Savajol, A.M. Benito, W.K. Maser, E. Munoz, M.T. Martinez, G.F. de la Fuente, A. Girard, and J.C. Ameline, Eur. Phys. J. A 18, 201 (2000).

${ }^{36}$ P.H. Tan, Y. Tang, C.Y. Hu, F. Li, Y.L. Wei, and H.M. Cheng, Phys. Rev. B 62, 5186 (2000).

${ }^{37}$ M.J. Peters, L.E. Mc Neil, J.P. Lu, and D. Kahn, Phys. Rev. B 61, 5939 (1999).

${ }^{38}$ M.J. Lopez, A. Rubio, J.A. Alonso, L.C. Qin, and S. Iijima, Phys. Rev. Lett. 86, 3056 (2001).

${ }^{39}$ J.-F. Colomer, L. Henrard, Ph. Lambin, and G. Van Tendeloo, Phys. Rev. B (to be published). 